$$
\text { CONF- } \underset{\substack{\text { UCRL-JC-121457 } \\ \text { PREPRINT }}}{9509 / 49--17}
$$

\title{
PHYSICS DESIGN AND SCALING OF RECIRCULATING INDUCTION ACCELERATORS: FROM BENCHTOP PROTOTYPES TO DRIVERS
}

\author{
J.J. Barnard, M.D. Cable, D.A. Callahan, T.J. Fessenden, ${ }^{+}$ \\ A. Friedman, D.P. Grote, D.L. Judd, S.M. Lund, M.A. Newton, JUV 051936 \\ W.M. Sharp, and S.S. Yu ${ }^{+}$ \\ Lawrence Livermore National Laboratory, University of California \\ P.O. Box 5508, L-440, Livermore California 94550, USA \\ ${ }^{+}$Lawrence Berkeley National Laboratory, University of Califormia \\ Berkeley California 94720, USA
}

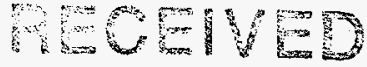

This paper was prepared for submittal to the Proceedings of the International Symposium On Heavy Ion Fusion

Princeton, New Jersey

September 6-9, 1995

February 6, 1996

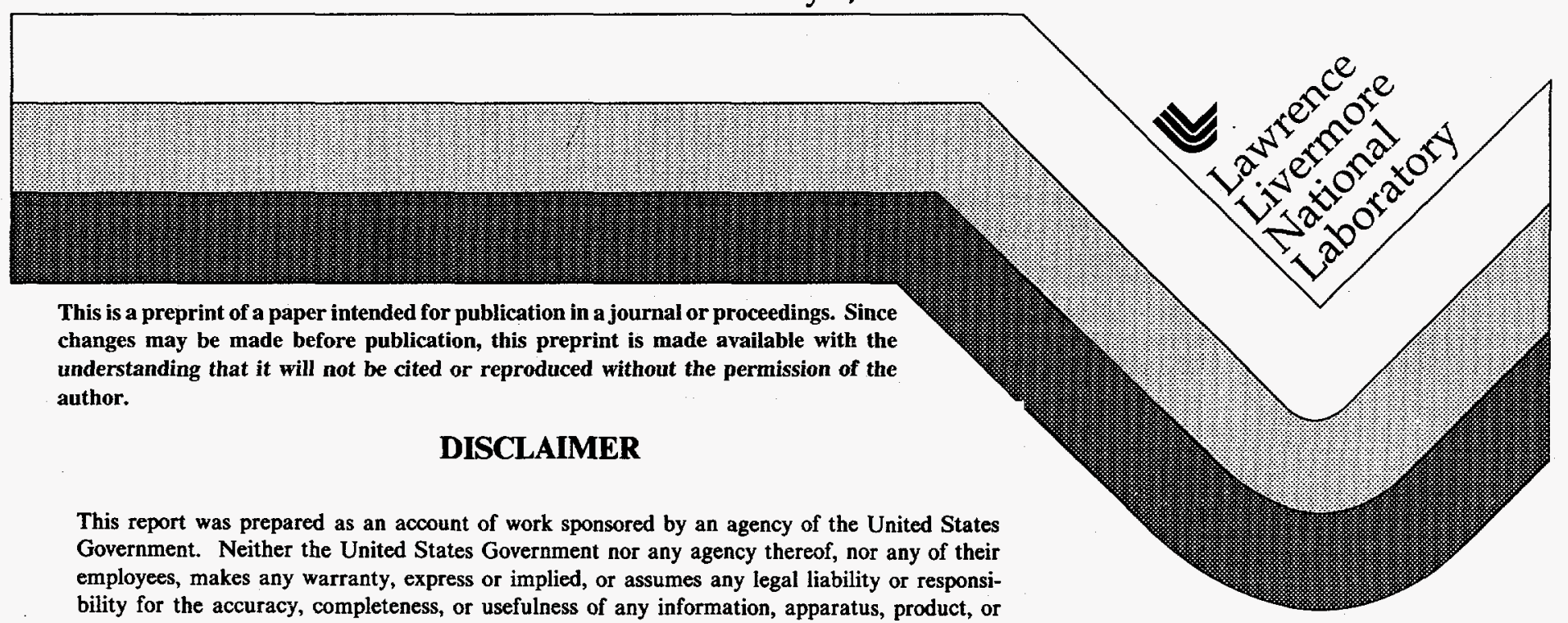
process disclosed, or represents that its use would not infringe privately owned rights. Reference herein to any specific commercial product, process, or service by trade name, trademark, manufacturer, or otherwise does not necessarily constitute or imply its endorsement, recommendation, or favoring by the United States Government or any agency thereof. The views and opinions of authors expressed herein do not necessarily state or reflect those of the United States Government or any agency thereof. 


\section{DISCLAIMER}

This document was prepared as an account of work sponsored by an agency of the United States Government. Neither the United States Government nor the University of California nor any of their employees, makes any warranty, express or implied, or assumes any legal liability of responsibility for the accuracy, completeness, or usefulness of any information, apparatus, product, or process disclosed, or represents that its use would not infringe privately owned rights. Reference herein to any specific commercial products, process, or service by trade name, trademark, manufacturer, or otherwise, does not necessarily constitute or imply its endorsement, recommendation, or favoring by the United States Government or the University of California. The views and opinions of authors expressed herein do not necessarily state or reflect those of the United States Government or the University of California, and shall not be used for advertising or product endorsement purposes. 


\section{DISCLAIMER}

Portions of this document may be illegible in electronic image products. Images are produced from the best available original document. 


\title{
PHYSICS DESIGN AND SCALING OF RECIRCULATING INDUCTION ACCELERATORS: FROM BENCHTOP PROTOTYPES TO DRIVERS *
}

\author{
J. J. Barnard ${ }^{\dagger}$, M. D. Cable ${ }^{\dagger}$, D. A. Callahan ${ }^{\dagger}$, T. J. Fessenden ${ }^{\ddagger}$, \\ A. Friedman ${ }^{\dagger}$, D. P. Grote ${ }^{\dagger}$, D. L. Judd ${ }^{\ddagger}$, S. M. Lund ${ }^{\dagger}$, \\ M. A. Newton ${ }^{\dagger}$, W. M. Sharp ${ }^{\dagger}$, and S. S. Yu ${ }^{\ddagger}$ \\ † Lawrence Livermore National Laboratory, Livermore, California, 94550 \\ $\ddagger$ Lawrence Berkeley National Laboratory, Berkeley, California, 94720
}

\begin{abstract}
Recirculating induction accelerators (recirculators) have been investigated as possible drivers for inertial fusion energy production because of their potential cost advantage over linear induction accelerators. Point designs were obtained in ref. [1], and many of the critical physics and technology issues that would need to be addressed were detailed. A collaboration (ref. [2]) involving Lawrence Livermore National Laboratory and Lawrence Berkeley National Laboratory researchers is now developing a small prototype recirculator in order to demonstrate an understanding of nearly all of the critical beam dynamics issues that have been raised in ref. [1] and subsequently.

We review the design equations for recirculators, (which have been incorporated into a Mathematicabased design code) and demonstrate how, by keeping crucial dimensionless quantities constant, a small prototype recirculator was designed which will simulate the essential beam physics of a driver. We further show how important physical quantities such as the sensitivity to errors of optical elements (in both field strength and placement), insertion/extraction, vacuum requirements, and emittance growth, scale from small-prototype to driver-size accelerator.
\end{abstract}

\section{INTRODUCTION}

Cost projections for inertial fusion power plants driven by beams of heavy ions accelerated by linear induction accelerators appear favorable. The purpose for studying recirculating induction accelerators (recirculators) is to provide an even lower cost alternative to the linear induction accelerator, the main-line U.S. approach to an Inertial Fusion Energy (IFE) driver. The cost advantage has been projected to occur [1], because in a recirculator, as in any circular accelerator, the focusing and accelerating components are used repeatedly so their total number can be reduced. Additionally since there is no longer a premium on a large accelerating gradient (which is necessary in a linac to minimize accelerator length and therefore cost), the accelerating gradient can be reduced, so that the accelerating induction cores can be individually reduced in size.

* Work performed under the auspices of the U.S. Department of Energy at LLNL under contract W-7405ENG-48 and at LBNL under contract DE-AC03-76SF00098. 
The principal elements of a recirculator are quadrupole magnets, to confine the beam transversely, ramped dipole magnets to bend the beam in a circle, and induction modules. In some designs, (as in ref. [1]), the bending field is separated into a DC component which is combined with the quadrupolar field in one superconducting combined function magnet, while a second ramped normal magnet provides a variable dipolar field, so that the total dipole ramps as the beam energy increases in time. The induction cores are composed of annular cylinders of ferromagnetic material which enable the pulse power system to generate an electric field across an accelerating gap for a time proportional to the cross-sectional area of the core. This longitudinal electric field provides beam acceleration, longitudinal confinement, and beam compression.

Although reuse of components by recirculation can be expected to lead to a lower cost, the reduction in cost is limited. The addition of bending magnets represents an additional cost over a linac. Further, since there is a need to compress and accelerate the ion beam simultaneously, the dynamic range in energy is limited to a factor of ten or so in each ring, requiring two to four rings to carry out the acceleration from $3 \mathrm{MeV}$ to $10 \mathrm{GeV}$, that is typical for a heavy ion fusion driver. The minimum scale of each ring is dictated by either the maximum practically achievable bending field or the need for high efficiency, requiring a minimization of the stored energy in the ramped bending field. (The stored energy scales as $B_{d}^{2} R \sim 1 / R$, where $B_{d}$ is the peak dipole magnetic field, and $R$ is the major radius of the machine.) The circumference of the lowest energy ring must be greater than about twice the initial beam length to accomodate the reset of magnetization of the induction cores. The greater the number of turns, the greater the cost savings due to reuse of components. The number of turns achievable, however, is limited by the minimum residual gas level of a practically achievable vacuum, which is the principal factor determining the amount of beam loss. Finally, because the induction cores are pulsed each turn (at up to $\sim 100 \mathrm{kHz}$ repetition rate) rather than each shot on target (at up to $\sim 10 \mathrm{~Hz}$ repetition rate, as in a linac) the pulse power must be more flexible to accomodate a pulse of high repetition rate that is changing its shape and duration over the course of about 100 turns. With all of these constraints, however, estimates in ref. [1] showed that a cost savings of about a factor of two can be achieved, and it is therefore a concept worth exploring in more detail. Some of the major parameters of one of the driver designs (the "C-design") from ref. [1] are listed in table 1.

In addition to transversely confining, accelerating, and longitudinally compressing the ion beams, recirculation adds the further requirement of bending the beams. There is also the previously discussed technological challenge of providing the temporally varying accelerating and confinement (or "ear") voltage pulses. Thus, a higher level of beam control is required than in a linac and a set of beam physics questions arises that has not been addressed in previous studies of transport of space-charge dominated beams.

The purpose of this paper is to demonstrate how a small ( $~ 14.4 \mathrm{~m}$ circumference) experimental prototype recirculator, termed the "small recirculator" (SR), can be designed to provide a scaled test of many of the beam physics and beam control questions that would arise on a large, driver-scale ( $\sim 2 \mathrm{~km}$ circumference) recirculator (DR). The SR is currently under development at Lawrence Livermore National Laboratory. A general review of the project is given by Friedman et al [2], a review of the mechanical design is given by 
Newton et al [3], and a review of the experimental results to date is given by Fessenden et al [4]. In section II, we identify the chain of reasoning which led to the preliminary design of an experiment. In section III through VI, we indicate how other aspects of the design scale from small experiment to driver. Also listed in table 1 are the main parameters of the SR. A layout of the SR is provided in ref. [2].

\section{OUTLINE OF SMALL RECIRCULATOR DESIGN}

In designing the SR there were three underlying principles which guided our decisions: Simplicity, low cost, and relevance to a driver. These principles were manifested by a set of practical, geometrical and driver-relevant constraints. Table 2 summarizes these constraints.

\section{Practical constraints}

Practical considerations dictated the general scale of the experiment. The circumference $C$ was chosen to be about $14 \mathrm{~m}$ in order to fit into a reasonable size experimental area $(\sim 5 \mathrm{~m}$ by $\sim 5 \mathrm{~m})$ yet be large enough to capture the essential physics. The pipe radius of about $3.5 \mathrm{~cm}$ was chosen to allow for a mean beam radius of about $1 \mathrm{~cm}$, which allocated enough clearance for displacements of the beam centroid arising from inevitable alignment and field errors, and to minimize non-linear image effects. The $1 \mathrm{~cm}$ beam radius, in turn, meant that relatively easy beam diagnostics and access to the beam were possible. The energy and current scales were set by choosing these quantities to be similar to experiments at Lawrence Berkeley National Laboratory (LBNL), the Single Beam Transport Experiments (SBTE) and Multiple Beam Experiment (MBE-4), which had energies in the 0.1-1 MeV range and currents in the 5-40 mA range, and which demonstrated the feasibility of transporting space charge dominated ion beams in linear induction accelerators. Potassium (atomic mass 39) was chosen to be the ion species because of the extensive experience gained at LBNL using zeolite sources. These sources are infused with potassium and liberate it when heated with an internal filament.

We have chosen to use permanent magnet quadrupoles for beam confinement, for their low cost and fidelity in simulating a DR. In the DR study superconducting electromagnetic quads were chosen to maintain high efficiency. The constant magnetic field of the permanent magnets best simulates this design and has the additional advantages of no power consumption and no heat load.

We have chosen ramped electric dipoles for our bending system, on the basis of simplicity, cost, and power requirements. Although ramped magnetic dipoles would have more faithfully simulated a driver, the ease of fabrication of two parallel electric dipole plates, together with the much lower power requirements and hence heat dissipation requirements of electric dipoles relative to magnetic dipoles, drove the decision toward the electric option. To accomodate the increasing energy of the beam the dipoles must ramp with time. If the beam acquires energy linearly with distance (i.e. quadratically with time) the dipole voltage must also ramp quadratically with time.

The scale and strength of the optical elements are dictated by both geometrical constraints, which are 
based on minimization of emittance growth due to non-linear fringe fields, and requirements that the physical regimes (based on dimensionless parameters) remain the same as a DR.

\section{Geometrical constraints on lattice elements}

There are two geometrical constraints which are used in the design. One limits the aspect ratio of the focusing and bending components of the recirculator, and the other relates the beam radius to the aperture. In particular, an LBNL scaling relation suggests (see for example ref. [5]) that the physical lengths of the quadrupoles be greater than $\sim 3 r_{p}$, where $r_{p}$ is the beam pipe radius through the quadrupole in order that the non-linear fields associated with the finite length of quadrupole be held to reasonably small values, to avoid emittance growth (i.e. transverse phase space dilution). We have assumed that a similar constraint also applies to the dipole, (where $r_{p}$ represents the half aperture through -the dipole.) Additionally one $r_{p}$ was reserved at each end of the dipole so that the non-linear end fields associated with the large change in potential between the dipole plates and the beam pipe threading the quad, would also be minimized. One additional length of $r_{p}$ at each end of the quad was reserved for flanges, diagnostics, and bellows. This allotment of longitudinal space in units of $r_{p}$ yields a ratio of half-lattice period $L$ to $r_{p}$ given by

$$
L / r_{p} \geq 10
$$

(10 $r_{p}$ is the sum of $3 r_{p}$ each for the quadrupole and dipole physical lengths and $4 r_{p}$ for the ends.) This ratio is nearly identical to that of the low energy ring of a driver (ref. [1]). This similarity occurs because the driver lattice was designed using very similar constraints on these dimensionless aspect ratios, except that fringe field considerations for the dipoles were replaced by considerations of return windings for the current magnets. A second scale consideration is that the ratio of $r_{p}$ to the maximum beam radius $a_{\text {max }}$ should be large enough to give adequate clearance between beam and pipe to allow for beam motion and to reduce the effects of image charges in creating non-linear fields for the beam to encounter. The LBNL scaling relation (see ref. [6]) suggests that

$$
r_{p}=1.25 a_{\max }+.01 \mathrm{~m}
$$

Additionally, in a recirculator, the velocity of the head will be somewhat smaller than the velocity of the tail in order to compress the bunch longitudinally. Thus, in a bend, the tail will have an equilibrium at a somewhat larger radius than the head, and so about $4 \mathrm{~mm}$ extra room is allowed for head-to-tail dispersion in the prototype. In a driver this is a negligible correction, because of the larger radius of curvature In the SR regime, this allotment of space corresponds to

$$
r_{p} / a_{\max } \cong 1.9
$$

In a driver, this ratio can be reduced to about 1.4 . 


\section{Constraints based on relevance to a driver}

The next set of design constraints ensures that the physical regime of the SR is the same as that of a DR. There are four dimensionless parameters which characterize the transverse focusing, space charge, thermal, centrifugal and inertial forces which must balance in order to maintain beam equilibrium. These are the "phase advance per lattice period" $\sigma_{0}$, the "depressed phase advance" $\sigma$, the perveance $K$, and the tune $\nu$. Before discussing these it is useful to examine the single particle equations of motion in the "smooth" approximation in which the focusing from the alternating gradient quadrupoles is approximated by a constant focusing field which has the same frequency of quasi-harmonic motion and the bend motion is approximated by a uniform bending field which gives the same angular deflection over one lattice period. Let the machine have average radius of curvature $\bar{\rho}$, and let a particle have radius $r$, and axial coordinate $s \equiv \bar{\rho} \theta$. Also, let the fractional difference between the particle momentum and the design momentum, be $\delta p / p$. Further, let $x \equiv r-\bar{\rho}$, and $y$ be the particle coordinate in the vertical direction. The equations of motion, through linear order in $k_{\beta_{0}} x, k_{\beta_{0}} y$, and $\delta p / p$ can then be written

$$
\begin{gathered}
x^{\prime \prime}=-k_{\beta 0 x}^{2}(x-\eta \delta p / p)+k_{s x}^{2}\left(x-x_{c}\right), \\
y^{\prime \prime}=-k_{\beta 0 y}^{2} y+k_{s y}^{2}\left(y-y_{c}\right) .
\end{gathered}
$$

Here primes indicate derivative with respect to $s, k_{\beta 0 x}^{2} \cong\left(\sigma_{0 \text { quad }}^{2}+\sigma_{0 b e n d x}^{2}\right) / 4 L^{2}$, $k_{\beta 0 y}^{2} \cong\left(\sigma_{0 \text { quad }}^{2}+\sigma_{0 b e n d y}^{2}\right) / 4 L^{2}$, where $\sigma_{0 q u a d}$ is the phase advance per lattice period from the quadrupoles alone, $\sigma_{0 b e n d x, y}$ is the contribution to the phase advance in the $x, y$ directions from the bends, and $x_{c}$, and $y_{c}$ are the centroid coordinates. Also in eqs. (4) and (5),

$$
\eta= \begin{cases}1 /\left(k_{\beta 0 x}^{2} \bar{\rho}\right) & \text { for magnetic bends, } \\ 2 /\left(k_{\beta 0 x}^{2} \bar{\rho}\right) & \text { for electric bends. }\end{cases}
$$

In eqs. (4) and (5) the transverse space charge force is represented by the linear force from a uniform charge density, which is given by

$$
k_{s x}^{2} \equiv \frac{2 K}{\left(a^{2}+a b\right)} ; \quad k_{s y}^{2} \equiv \frac{2 K}{\left(b^{2}+a b\right)} .
$$

Here, $a$ and $b$ are the ellipse semi-axes of the beam envelope in the $x$ and $y$ directions respectively, and $K$ is the generalized perveance defined shortly.

Although eqs. (4) through (7) contain much of the physics required to obtain a zeroth order design of a DR or SR, some notable physics is absent. In addition to omission of effects due to the finite length of the elements, image charges have been neglected and the paraxial approximation has been employed.

The first terms in eqs. 4 and 5 represent the effects of the external focusing. The phase advance per lattice period $\sigma_{0} \equiv 2 L k_{\beta_{0}}$ is a measure of the strength of the confining force relative to the inertia of the particles. An accurate approximate calculation of $\sigma_{0 q u a d}$ has been carried out in ref. [7]:

$$
\cos \left(\sigma_{0 q u a d}\right) \cong 1-\frac{1}{6}\left(3-2 \eta_{q}\right) \eta_{q}^{2}\left(\frac{B^{\prime}}{[B \rho]}\right)^{2} L^{4}
$$


Here $\eta_{q} \equiv l_{q e f f} / L$ is the occupancy of the quad, where $l_{q e f f}$ is the effective length of the quad, $B^{\prime}$ is the quadrupole gradient, $[B \rho] \equiv p / q e$ is the ion rigidity, $p$ is the ion design momentum, $q$ is the charge state of the ion, $e$ is the proton charge.

For the purposes of scaling it is often more useful to examine the "thin" lens limit (in which $\eta_{q} \rightarrow 0$ and $B^{\prime} \rightarrow \infty$, while the product remains finite), in which case $\sigma_{0}$ is given by (ref. 19).

$$
\sigma_{0 q u a d} \cong \frac{\eta_{q} L^{2} B^{\prime}}{[B \rho]}
$$

In our design code we use the more exact representation in eq. (8), but we will use the smooth approximation for illustration of simple scaling relations.

Although not a significant source of phase advance in a DR, the dipoles have an additional.focusing force arising from the change in longitudinal velocity, which arises from the voltage drop across the plates. For ideal plates of equal length (in which fringe fields are neglected), the contribution to the phase advance is:

$$
\sigma_{0 b e n d x} \cong \frac{2 L}{\eta_{d} \bar{\rho}} .
$$

Here $\eta_{d}$ is the occupancy of the bend. Magnetic sector bends give approximately the same contribution to the focusing (albeit for different reasons). In the idealized case, the electric bends contribute no phase advance in the $y$ direction, but there will be a contribution in a real fringe field. Careful shaping of the plates (ref. [8]), using the 3D code WARP has led to a design in which the focusing is equal in both $x$ and $y$. The plates are curved about the $z$ axis to provide focusing in $y$ and have ridges on the top and bottom edges to minimize the sextupole content.

Studies on SBTE (ref. [10]) have shown that beam instabilities and beam loss sets in when

$$
\sigma_{0}>\sim 88^{\circ}
$$

To allow a margin of safety, we adopted the constraint that $\sigma_{0}$ be less than $80^{\circ}$, an appropriate constraint for both the DR and SR.

The term in eqns. (4) and (5) proportional to $k_{s x}^{2}$ represents the outward force of the beam space charge which is proportional to the perveance $K$, and is a measure of the space charge potential energy relative to kinetic energy. In terms of beam quantities (and in the non-relativistic limit):

$$
K \equiv \frac{2 q e}{4 \pi \epsilon_{o} A m_{a m u} c^{3}} \frac{I}{\beta^{3}} .
$$

Here, $A$ is the atomic mass of the beam ions in amu, $m_{a m u}$ is atomic mass unit, $\beta c$ is the ion velocity, $I$ is the beam current, and $\epsilon_{0}$ is the permitivity of free space. The SR will necessarily have a much lower current than a DR, but the much smaller velocity allows the SR to be in a regime where the dynamic effects of space charge are comparable to a driver. Requiring $K \sim 10^{-4}$ to $10^{-3}$ (i.e. in the range of a driver) places another constraint on the SR design. 
The "normalized emittance" $\epsilon_{n x} \equiv 4 \beta\left(\left\langle x^{2}\right\rangle\langle x\rangle^{2}-\left\langle x x^{\prime}\right\rangle^{2}\right)^{1 / 2}$ is a measure of the transverse phase space of the beam and is a conserved quantity in the presence of linear, uncoupled focusing, bending and space charge forces. In order to focus onto a spot at the output end of the accelerator, the normalized emittance must remain small. This, coupled with the requirement of high particle current leads to a situation where the thermal forces are generally much smaller than the space charge forces during the acceleration. In such space charge dominated beams the focusing force is nearly compensated by the outward force of space charge. The approximate relationship between these forces is described by the "envelope equation" in the limit of continuous focusing:

$$
\frac{\mathrm{d}^{2} a}{\mathrm{~d} z^{2}}=-\left(\frac{\sigma_{0}}{2 L}\right)^{2} a+\frac{K}{a}+\frac{\epsilon_{N}^{2}}{\beta^{2} a^{3}} .
$$

Here, $a$ represents the mean beam radius. The depressed phase advance $\sigma$ represents the change in phase in a lattice period of quasi-harmonic oscillation of an ion within the beam. Approximately, $\sigma^{2} \cong \sigma_{0}^{2}-4 L^{2} K / a^{2}$ and, from eq. 13 for a beam in equilibrium,

$$
\sigma \cong 2 \epsilon_{N} L / \beta a^{2}
$$

which indicates that the difference between the focusing and space charge forces is balanced by thermal forces. So to be in the same physical regime as a driver

$$
\sigma / \sigma_{0}<<1
$$

Another requirement to verify driver physics is to examine whether the growth in the normalized emittance is acceptable in the presence of the combined bending and space charge forces. One measure of the radius of curvature of the bend is the number of betatron wavelengths per circumference $\nu$ the so-called "tune". In both the SR and DR, the focusing magnetic field is fixed, so that as the beam gains energy the phase advance decreases. In a conventional synchrotron, the quadrupolar focusing field increases with the dipole field so that the phase advance remains fixed. In order to ensure that imperfections are not encountered at the same betatron phase each orbit, the circumference must be chosen not to be an integral or half-integral number of betatron wavelengths $\lambda_{\beta}$. In the recirculator, the tune changes on each orbit, so that at times during the acceleration sequence $\nu$ will be an integer. When the change in the number of betatron periods $\gtrsim>\sim 1$ the change in phase will be greater than or order $2 \pi$ so transverse kicks from field imperfections will add essentially incoherently. Thus, to be in the regime where the beam will rapidly pass through resonances, we require $\nu>>1$. However, as will be discussed in section IV steering corrections will be made at least once per lap in the SR, removing the possibility of resonance instability. In addition, the ratio of displacement of an off momentum particle relative to the beam radius gives another measure of the strength of the bend forces. Eqs. (4) and (6) imply that this ratio will be proportional to $\delta p / p /\left(k_{\beta_{0}} a \nu\right)$ and since $\delta p / p$ will be larger for the SR than for the DR and as will be shown $\nu$ will be smaller for the SR, the SR will provide a more extreme laboratory for understanding the transport of space-charge dominated beams in an accelerator with bends. 
The SR should also demonstrate the other major functions of the DR: energy gain and bunchlength compression. We require an energy gain $E_{f} / E_{i} \gtrsim 4$, where $E_{f}$ is the final energy and $E_{i}$ is the initial energy, and a bunch length compression $l_{f} / l_{i} \lesssim 1 / 2$ where $l_{f}$ is the final bunch length and $l_{i}$ is the initial bunch length. Finally we wish to accomplish all of this at high repetition rate, so the ion lap time should be less than about $20 \mu \mathrm{s}$.

\section{Application of Constraints to the Small Recirculator}

Given the above set of constraints, many of the features of our design are determined. Since the minimum pipe radius has been chosen to be about $3.5 \mathrm{~cm}$, then by eqn. $1, L \gtrsim 35 \mathrm{~cm}$ (and was chosen to be $36 \mathrm{~cm}$ ). Since $C$ is constrained to fit the available experimental area, the number of half-lattice periods $n_{h l p}$ is also constrained, and is the nearest even number satisfying: $n_{h l p}=C / L$, which in the case of the $\mathrm{SR} \sim 40$. The number of betatron periods is then also fixed: $\nu=C / \lambda_{\beta}=(C / L)\left(\sigma_{0} / 4 \pi\right) \leq 5$.

The perveance is estimated by equating the first and second terms in eq. 13 ,

$$
K \cong \frac{1}{4}\left(\frac{r_{p}}{L}\right)^{2}\left(\frac{a_{\max }}{r_{p}}\right)^{2}\left(\frac{a}{a_{\max }}\right)^{2} \sigma_{0}^{2} \leq 7.4 \times 10^{-4}
$$

Here, we have also used the constraint that the alternating gradient produces a "flutter motion" which yields a maximum beam radius $a_{\max }$ greater than the average beam radius $a$ according to $a_{\max } / a \cong$ $\left(2\left(1-\cos \sigma_{0}\right)\right)^{1 / 2} / 4 \cong 1+\sigma_{0} / 4$ (ref. [7]), which is approximately 1.35 for $\sigma_{0}=1.4$. Not surprisingly as mentioned above, the perveance for $\mathrm{DR}$ and SR are of the same order. The initial ratio of $\sigma / \sigma_{0}$ becomes

$$
\frac{\sigma}{\sigma_{0}} \cong\left(\frac{2 \sqrt{2}}{\sigma_{0}}\right)\left(\frac{k T_{\perp}}{E_{i}}\right)^{1 / 2}\left(\frac{r_{p}}{a_{\max }}\right)\left(\frac{a_{\max }}{a}\right)\left(\frac{L}{r_{p}}\right) \gtrsim 0.20 .
$$

Here, $k T_{\perp}=1 \mathrm{eV}$ and $E_{i}=80 \mathrm{keV}$ was assumed for the final inequality. Although somewhat smaller beam temperatures $(\sim .1 \mathrm{eV})$ are anticipated as they leave the source, we have expect that by the time the beam enters the ring the beam temperature will have increased to $\sim 1 \mathrm{eV}$, because of the matching and insertion process. Injection energies much less than our design of $80 \mathrm{keV}$ would start to run into difficulties maintaining a space charge dominated beam.

Demonstrating a factor of four increase in the ion energy requires a final energy of $320 \mathrm{keV}$. The tune depression $\sigma / \sigma_{0}$, the dipole-voltage, repetition rate, quadrupole field and pulse duration, are then all essentially determined. The total dipole voltage drop across the plates $V_{d}$ is determined by equating the average bend force to the centrifugal force:

$$
V_{d}=\left(\frac{E_{\text {final }}}{q e}\right)\left(\frac{8 \pi r_{p}}{C \eta_{d}}\right)=67 \mathrm{kV}\left(\frac{r_{p}}{3.5 \mathrm{~cm}}\right)\left(\frac{E_{\text {final }}}{320 \mathrm{keV}}\right)\left(\frac{14 \mathrm{~m}}{C}\right)\left(\frac{.3}{\eta_{d}}\right)
$$

Here $\eta_{d}$ is the fraction of the lattice period occupied by the physical dipole. The final lap time $t_{\text {lap final }}$ :

$$
t_{\text {lap final }}=\left(\frac{C}{\beta c}\right) \cong 11 \mu \mathrm{s}\left(\frac{C}{14 \mathrm{~m}}\right)\left(\frac{320 \mathrm{keV}}{E_{f i n a !}}\right)^{1 / 2}\left(\frac{A}{39}\right)^{1 / 2}
$$


This quantitty $t_{\text {Iap final }}$ determines the maximum repetition rate of the induction cell pulsers, which is thus about $100 \mathrm{kHz}$. The quadrupole field $B_{q}$ can be estimated (in the thin lens approximation) by

$$
B_{q} \cong\left(\frac{r_{p}}{L}\right)\left(\frac{[B \rho] \sigma_{0}}{\eta_{q} L}\right) \cong 0.33 \mathrm{~T}\left(\frac{10}{L / r_{p}}\right)\left(\frac{E_{i n i t i a l}}{80 \mathrm{keV}}\right)^{1 / 2}\left(\frac{A}{39}\right)^{1 / 2}\left(\frac{\sigma_{0}}{1.4}\right)\left(\frac{.36 \mathrm{~m}}{L}\right)
$$

To allow time to reset the magnetization in the induction cores, the maximum (and also initial) pulse duration $t_{p \text { initial satisfies }}$

$$
t_{p_{\text {initial }}} \cong \frac{1}{4} t_{\text {lap initial }} \cong 4 \mu \mathrm{s}\left(\frac{C}{14 \mathrm{~m}}\right)\left(\frac{80 \mathrm{keV}}{E_{\text {initial }}}\right)^{1 / 2}\left(\frac{A}{39}\right)^{1 / 2}
$$

The beam current of 2 to $8 \mathrm{~mA}$ follows from the energy and perveance. The design that we have obtained thus inherently models much of the dynamics of a $D R$, and the timescales are such that the articulation of the pulse power will also be similar to the timescales of a DR, (although at substantially lower voltages).

\section{Acceleration and Ears}

For engineering simplicity, we elect to have one set of parallel FET switches (rather than several sets in series as in the DR) control the voltage modulation on the induction cores. This limits the voltage on each cell to approximately $500 \mathrm{~V}$. The number of induction cores $n_{\text {core }}$ on the SR is 34 . (The other 6 half-lattice periods are reserved for the insertion/extraction section and an additional extraction section and have no cores). To achieve the minimum voltage increment of $240 \mathrm{kV}$, a minimum number of laps $n_{\text {lap }}$ $=14$ is required. Detailed accelerating waveforms, using the self-similar current replication procedure of Kim and Smith (ref. [17]), have been calculated using the REC code, which are then used as inputs to the fluid/envelope code CIRCE and the 3D PIC code WARP (see ref. [18] for details).

In order to calculate the magnitude of the confining electric fields, we use the longitudinal equation of motion in the long wavelength regime (see, e.g. ref. [14]; also, ref. [15] for a more exact treatment):

$$
\frac{\mathrm{d} v}{\mathrm{~d} t} \cong \frac{-g q e}{A m_{a m u}} \frac{\partial \lambda}{\partial \zeta}+\frac{q e}{A m_{a m u}} E_{\text {ext }} .
$$

Here, $\zeta=s-\beta c t, E_{\text {ext }}$ is the field applied in the acceleration gaps (both acceleration and ear fields), and $g=\ln \left[r_{p} / a\right] / 2 \pi \epsilon_{0}$ is the longitudinal space charge factor, where $\epsilon_{0}$ is the permittivity of free space, and $\beta c$ is the beam velocity at the center of the pulse. For a quadratic line-charge density profile, with an end that falls like $\lambda=\lambda_{0}\left(2 \zeta / l_{\text {end }}-\left(\zeta / l_{\text {end }}\right)^{2}\right)$, the maximum derivative $\mathrm{d} \lambda / \mathrm{d} \zeta$ occurs at the very tip, $(\zeta=0)$, where its value is $2 \lambda_{0} / l_{\text {end }}$. Furthermore, at the tip, the beam is emittance dominated, and so by eq. 13 , the mean beam radius $a \cong\left(2 L \epsilon_{n} / \beta \sigma_{0}\right)^{1 / 2}$. For a $0.5 \mathrm{~m}(0.4 \mu \mathrm{s})$ beam end at the end of the acceleration sequence $\lambda_{0} \cong 7.2 \times 10^{-3} \mu \mathrm{C} / \mathrm{m}$, and $\epsilon_{n} \cong 0.2 \mathrm{~mm}$-mrad. The maximum average confining "ear" field $E_{\text {earmax }}$ is then $\sim 840 \mathrm{~V} / \mathrm{m}$, which requires a maximum average gap voltage $V_{\text {earmax }}=E_{\text {earmax }} C / n_{\text {core }}$ of $350 \mathrm{~V}$, which is comparable to the acceleration voltage. In a $\mathrm{DR}$ the acceleration and confining voltage pulses are also comparable to each other, although the magnitudes of the voltages are larger (up to $85 \mathrm{kV}$ ) in the DR.

\section{TRANSVERSE EMITTANCE GROWTH}


The key to successful ignition of a capsule in an IFE driver is the ability to deliver the multiple ion beams onto small spots on the target. In order to achieve that goal, the normalized beam emittance must remain small ( $\lesssim 8 \mathrm{~mm} \mathrm{mrad).} \mathrm{Simulations} \mathrm{and} \mathrm{theory} \mathrm{to} \mathrm{date} \mathrm{have} \mathrm{revealed} \mathrm{only} \mathrm{one} \mathrm{source} \mathrm{of} \mathrm{emittance} \mathrm{growth}$ which has been observed, and which is difficult to avoid. That is, there appears to be a jump in emittance that occurs when a space charge dominated beam with a spread in longitudinal velocity makes a transition from a straight transport section to a bent one (or vice versa).

The beam is initially created from a circular hot plate and is transformed into an elliptical beam as it passes through a matching and transport section before it enters into the ring. When it enters the ring, it can evolve towards a new equilibrium where the average centrifugal force balances the average bending force. Since particles with larger longitudinal velocities will have larger equilibrium radii with respect to the center of the ring, the orbit of a particle with a momentum differing by $\Delta p$ from the design momentum $p$ by an amount $\Delta p$ will be displaced on average by an amount $\eta \delta p / p$, according to eq. 4 . When the beam enters the bend, the initial beam will broaden by an amount proportional to the rms average of the momentum distribution. An envelope mismatch will occur, and any non-linear space charge forces will eventually turn the mismatch into emittance growth. By equating the initial transverse energy to the final transverse energy and assuming equilibria in both initial and final states it is straightforward to calculate the rise of emittance (ref. [11]). The calculation in ref. 11 assumed the beam was distributed with a uniform elliptical charge density, and was therefore not completely self-consistent, but it nevertheless gave reasonable agreement to simulations. For small changes in emittance from the initial values, the change in the emittance was calculated to be

$$
\epsilon_{n x}^{2}-\epsilon_{n x 0}^{2} \cong \frac{\beta^{2}}{L^{2}} \frac{\sigma_{0}^{4}\left(2 \sigma_{0}^{2}+\sigma^{2}\right)}{\sigma^{2}\left(\sigma_{0}^{2}+\sigma^{2}\right)} a^{2} \eta^{2}\left\langle\delta p^{2} / p^{2}\right\rangle
$$

and

$$
\epsilon_{n y_{i}}^{2}-\epsilon_{n y 0}^{2} \cong \frac{\beta^{2}}{L^{2}} \frac{\sigma_{0}^{6}}{\sigma^{2}\left(\sigma_{0}^{2}+\sigma^{2}\right)} a^{2} \eta^{2}\left\langle\delta p^{2} / p^{2}\right\rangle
$$

If we assume $\left\langle\delta p^{2} / p^{2}\right\rangle^{1 / 2} \cong 2.5 \times 10^{-3}, \eta \cong 0.24 \mathrm{~m}, \sigma_{0} \cong 1.4, \sigma \cong 0.28, a \cong 0.01 \mathrm{~m}$ and $\epsilon_{n x 0} \cong 0.1 \mathrm{~mm}-$ mrad, then after insertion into the ring the $x$ and $y$ emittance should rise to approximately 0.29 and $0.28 \mathrm{~mm}$ mrad respectively. (The formulas above assume small changes in emittance for validity; a numerical solution of the energy conservation equations yields a final emittance of 0.175 and $0.164 \mathrm{~mm}-\mathrm{mrad}$, respectively, very close to the results obtained from the 3D WARP simulations). In a DR, $\bar{\rho}$ is larger (by a factor up to a $100)$ and $\delta p / p$ is smaller by a factor of 10 or more, so that for the initial injection into the ring the change in emittance is very small. However, in the racetrack designs of ref. [1], there are long straight sections, which could create mismatches twice per lap, providing a gradual heating of the beam. In ref. [11], this process was estimated to lead to nearly a doubling of the normalized emittance, about equal to the allotted emittance budget for the ring.

IV. ERROR SCALING 
When the acceleration gradient is sufficiently rapid, and the focusing field remains constant, the beam centroid responds to misalignments as if they were statistically independent. The transverse energy of the beam centroid statistically increases linearly with $s$ and so the evolution of the centroid position $x_{c}$ can be described roughly as a "random walk." The two major errors which contribute to the walk in $x$ are dipole strength errors and quadrupole displacement errors, while in $y$ the two largest errors are dipole rotation errors and also quad displacement errors. A rough estimate of the displacements for intervals over which the energy is nearly constant is given by (see e.g. Sharp et al, ref. [16]);

$$
\begin{gathered}
x_{c m a x}^{2} \cong n_{l a p} n_{h l p}\left[\left(\frac{16 \pi^{2} L^{2}}{n_{h l p}^{2}}\right)\left\langle\frac{\delta E_{i}^{2}}{\sigma_{0 x}^{2} E_{d}^{2}}\right\rangle+4\left\langle\frac{f_{1}^{2} \sigma_{0 q u a d}^{2} \delta_{q i}^{2}}{\sigma_{0 x}^{2}}\right)\right] \\
y_{c m a x}^{2} \cong n_{l a p} n_{h l p}\left[\left(\frac{16 \pi^{2} L^{2}}{n_{h l p}^{2}}\right)\left\langle\frac{\delta \theta_{i}^{2}}{\sigma_{0 y}^{2}}\right\rangle+4\left\langle\frac{f_{1}^{2} \sigma_{0 q u a d}^{2} \delta_{q i}^{2}}{\sigma_{0 y}^{2}}\right\rangle\right] .
\end{gathered}
$$

Here, $\delta E_{i}$ is the dipole field strength error, $\delta \theta_{i}$ is the dipole rotational error, $\delta_{q i}$ is the quadrupole displacement error, $f_{1}$ is a factor of order unity, and \langle\rangle indicates a statistical average. Although these relations are useful guides, simulations using the envelope/fluid code CIRCE (ref. [16]) have given more definitive error tolerances. For a maximum displacement of $1 \mathrm{~cm}$ after 1 lap, and assuming equal contributions from the two error sources in each direction, the simulations imply that a $250 \mu \mathrm{m}$ rms quadrupole alignment error, a $1 \%$ dipole field strength error, and a $0.1^{\circ}$ dipole rotation error are allowed. Since achievement of greater precision can entail greater cost, we have elected to use steering to keep the beam near the axis. This steering will use information from previous shots gained through non-intercepting capacitive probes (see ref. [4]). We plan to correct by making slight alterations to the dipole voltage, and also by using the capacitive probes as steering dipoles. For a driver, electric field strength errors in eq. (24) are replaced by magnetic field strength errors, and since $n_{h l p}$ and $L$ increase by similar factors, corrections should be made after a similar number of half-lattice periods to obtain similar tolerance requirements. We note that when corrections are made at least once per lap, resonance stability questions are not pertinent since betatron phase information is lost during correction.

\section{VACUUM REQUIREMENTS}

Because the beam path in a recirculator is much longer than in a linac, recirculators have more stringent vacuum requirements. The requirement on the background density is set by stripping (and neutralization) by the residual gas:

$$
\left.n_{\text {residual }} \cong \frac{1}{\sigma_{s} n_{\text {lap }} C} \frac{\delta n_{b}}{n_{b}}\right|_{\text {strip }}
$$

For singly ionized potassium in the $80-320 \mathrm{keV}$ energy range, measured combined cross sections $\sigma_{s}$ (ref. [12]) of stripping and neutralization by atmospheric gasses are of order $0.5-1.0 \times 10^{-15} \mathrm{~cm}^{2}$; in this regime the cross section is increasing with energy. For the SR design, a beam loss $\left.\left(\delta n_{b} / n_{b}\right)\right|_{s t r i p}$ of $1 \%$ is allowed, and with the nominal number of laps set to 15 , this suggests a maximum permissible residual gas of about $10^{-8}$ Torr. In the DR, the path length is increased from $200 \mathrm{~m}$ in the SR to $200 \mathrm{~km}$ in the High Energy 
Ring. The cross-section for stripping, however, is in a regime which is scaling inversely with energy, and for atomic mass 200 the cross-section is of order $10^{-16} \mathrm{~cm}^{2}$, requiring a residual gas level less than about $10^{-10}$ Torr. Additionally, beam-induced wall desorption by ionized residual gas particles, and beam-beam charge exchange are predicted to be significant contributors to beam loss in the the DR, but not in the SR.

\section{INSERTION/EXTRACTION}

Insertion of the beam into the ring and extraction from it is another area to be explored in the SR. Because the normal alternating gradient focusing must be present on a normal lap (i.e. not an extraction or insertion lap) and because the quadrupoles occupy a significant fraction of the half lattice period, it appears to be prohibitively difficult to insert or extract a beam through a normal quad and still be able to clear the subsequent quad. In a DR, the approach taken in ref. [1] was to extract the. beam through a number ( 14) of "Panofsky" type, wide aperture quads. The orbit would be driven to large amplitude in about 0.37 of a betatron period (to minimize energy dispersion), using a similar number of "kicker bends" to excite essentially a large betatron oscillation, to the point where the beam can clear the adjacent normal quadrupole. In the SR, the lower energy and the smaller radius of curvature of the ring require smaller kicker fields, allowing insertion and extraction to occur through one wide-aperture permanent magnet. (See ref. [13] for detailed calculations of its design). The experiment will test whether the abrupt manipulations required on insertion and extraction can be carried out with an acceptably small increase in emittance.

\section{CONCLUSION}

Induction recirculators promise a lower cost driver for inertial fusion energy. In this paper, we have described the considerations leading to the physics design of a scaled prototype experiment, which will, by construction, test our understanding of beam transport of intense, space charge dominated ion beams in driver scale circular accelerators.

\section{REFERENCES}

${ }^{1}$ J.J. Barnard, A.L Brooks, J.P. Clay, F.J. Deadrick, L.V. Griffith, A.R. Harvey, D.L. Judd, H.C. Kirbie, V.K. Neil, M.A. Newton, A.C. Paul, L.L. Reginato, G.E. Russell, W.M. Sharp, H.D. Shay, J.H. Wilson, and S.S. Yu, UCRL-LR-108095, "Recirculating Induction Accelerators as Drivers for Heavy Ion Fusion," Lawrence Livermore National Laboratory (1991). Also J.J. Barnard, F. Deadrick, A. Friedman, D.P. Grote, L.V. Griffith, H.C. Kirbie, V.K. Neil, M.A. Newton, A.C. Paul, W.M. Sharp, H.D. Shay, R.O. Bangerter, A. Faltens, C.G. Fong, D.L. Judd, E.P. Lee, L.L. Reginato, S.S. Yu, and T.F. Godlove, Physics of Fluids B: Plasma Physics, 5, 2698 (1993);

${ }^{2}$ A. Friedman, J.J. Barnard, M.D. Cable, D.A. Callahan, F.J. Deadrick, D.P. Grote, V.P. Karpenko, H.C. Kirbie, B.G. Logan, S.M. Lund, L.A. Nattrass, M.B. Nelson, M.A. Newton, T.C. Sangster, W.M. Sharp, T.J. Fessenden, D.L. Judd, S. Eylon, H.S. Hopkins, D.B. Longinotti, "Recirculating Induction Accelerators for Inertial Fusion: Prospects and Status," this volume. 
${ }^{3}$ M.A. Newton, F.J. Deadrick, R.L.Hanks, H.C. Kirbie, V.P. Karpenko, L.A. Nattrass, “Engineering Development for a Small-Scale Recirculator Experiment," this volume.

${ }^{4}$ T.J. Fessenden, J.J. Barnard, M.D. Cable, F.J. Deadrick, M.B. Nelson, M.A. Newton, T.C. Sangster, S. Eylon, H.S. Hopkins, "Intense Heavy-Ion Beam Transport with Electric and Magnetic Quadrupoles," this volume.

5 W.M. Fawley, L.J. Laslett, C.M. Celata, A. Faltens, I. Haber, "Simulation Studies of Space-ChargeDominated Beam Transport in Large Aperture Ratio Quadrupoles," Proceedings of the 1993 Particle Accelerator Conference, Washington, D.C.,1, 724 (1993).

${ }^{6}$ P. Seidl and A. Faltens, "Electrostatic Quadrupoles for Heavy Ion Fusion," Proceedings of the 1993 Particle Accelerator Conference, Washington, D.C.,1, 721 (1993).

7 E.P. Lee, T.J. Fessenden, J. Laslett, IEEE Transactions on Nuclear Science, NS-32 2489 (1985).

${ }^{8}$ D.P. Grote and A. Friedman, "Three Dimensional Simulations of High Current Beams in Induction Accelerators with WARP 3D," this volume.

${ }^{9}$ A. Friedman, D.A. Callahan, D.P. Grote, I. Haber, A.B. Langdon, and S.M. Lund, "What We've Learned from 3-D and R,Z Intense-Beam Simulations Using the WARP Code," Nuovo Cimento A, 107, 1649 (1994)

${ }^{10}$ Tiefenback, M.G. Space-Charge Limits on the Transport of Ion Beams in a Long Alternating Gradient System," Ph.D thesis, Univ. of California, Berkeley, LBL-22465 (1986).

11 J.J. Barnard, H.D.Shay, S.S. Yu, A. Friedman; and D.P. Grote, "Emittance Growth in Heavy-Ion Recirculators," 1992 Linear Accelerator Conference Proceedings 1992 August 24-28, Ottawa, Ontario, Canada, C.R. Hoffman, ed. AECL 10728 (AECL Research, Chalk River, Canada) 229 (1992). (Note, that eqs. 11 and 12 in this reference have errors in the numerical coefficients and should be replaced by eqs. 23 of the text).

${ }^{12}$ H.H. Lo, and W.L. Fite, Atomic Data, 1, 305 (1970)

${ }^{13}$ S. M. Lund and K. Halbach, "Permanent Magnet Systems for Charged Particle Optics," this volume.

${ }^{14}$ For example, M. Reiser, "Theory and Design of Charged Particle Beams," [John Wiley and Sons, NY], Chapter 6 (1994).

${ }^{15}$ W.M. Sharp, A. Friedman and D.P. Grote, "Effects of Space Charge in Beams for Heavy Ion Fusion," this volume.

${ }^{16}$ W.M. Sharp, J.J. Barnard, and S.S. Yu, "Steering Algorithms for a Heavy-Ion Recirculating Accelerator," Proceedings of the Ninth International Conference on High-Power Particle Beams, Washington D.C. May 25-29, 1992.

.17 C.H. Kim and L. Smith, "A Design Procedure for Acceleration and Bunching in an Ion Induction Linac," Particle Accelerators, 85, 101 (1986).

18 W.M. Sharp, J.J. Barnard, A. Friedman, D.P. Grote, S.M. Lund, M.A. Newton, T.J. Fessenden, and S.S. $\mathrm{Yu}$, "Numerical Modeling of a Small Recirculating Induction Accelerator for Heavy-Ion Fusion," Proceedings 
of the Fourth European Particle Accelerator Conference, London, 27 June-1 July 1994 2, 1397 (1994). ${ }^{19}$ Reiser, Martin, "Theory and Design of Charged Particle Beams" [Wiley, Interscience, NY] (eq. 3.362b), (1994). 
Table 1. Summary of Parameters for the Small and Driver Recirculators

\begin{tabular}{|c|c|c|c|c|}
\hline & $\underline{\mathrm{SR}} *$ & $\underline{\text { LER }^{*}}$ & $\underline{\text { MER }^{*}}$ & $\underline{\text { HER }^{*}}$ \\
\hline Ion Energy (MeV) & $0.08-0.32$ & $3-50$ & $50-1000$ & $10^{3}-10^{4}$ \\
\hline Pulse Duration ( $\mu \mathrm{s})$ & $4-1$ & $200-30$ & $30-2.5$ & $2.5-.25$ \\
\hline Circumference (m) & 14 & 700 & 921 & 1976 \\
\hline Current/Beam (A) & $2-8 \times 10^{-3}$ & $0.5-3.3$ & $3.3-40$ & $40-400$ \\
\hline No. of Beams & 1 & 4 & 4 & 4 \\
\hline No. of Corelines & 1 & 1 & 1 & 1 \\
\hline No. of Laps & 15 & 100 & 100 & 100 \\
\hline Pipe Radius (cm) & 3.5 & 7.8 & 6.4 & 6.1 \\
\hline Lattice Half Period (m) & 0.36 & 0.85 & 1.56 & 3.51 \\
\hline Vacuum (Torr) & $1 \times 10^{-8}$ & $5 \times 10^{-11}$ & $5 \times 10^{-11}$ & $2 \times 10^{-10}$ \\
\hline \multicolumn{5}{|l|}{ Induction Modules: } \\
\hline Inn. radius (m) & .163 & .313 & .255 & .243 \\
\hline Out. radius (m) & .211 & .455 & .554 & .363 \\
\hline Length $(\mathrm{m})$ & .085 & .403 & .837 & .895 \\
\hline No. of Cores & 34 & 785 & 551 & 1068 \\
\hline Cell voltage $(\mathrm{kV})$ & 0.50 & 0.60 & 17.4 & 85 \\
\hline \multicolumn{5}{|c|}{ Bends (Ramped Magnetic or Electric Dipoles): } \\
\hline Effective Length (m) & 0.13 & 0.15 & 0.41 & 1.15 \\
\hline No. of Bends & 40 & 2680 & 1796 & 1919 \\
\hline Max. Field & $910 \mathrm{kV} / \mathrm{m}$ & $0.90 \mathrm{~T}$ & $0.85 \mathrm{~T}$ & $0.81 \mathrm{~T}$ \\
\hline Max. Eff. Field & $910 \mathrm{kV} / \mathrm{m}$ & $0.90 \mathrm{~T}$ & $2.2 \mathrm{~T}$ & $2.35 \mathrm{~T}$ \\
\hline \multicolumn{5}{|l|}{ Quadrupoles/D.C. Dipoles: } \\
\hline Length (Eff. Length) (m) & $0.10(0.10)$ & $.47(.23)$ & $.92(.73)$ & $1.94(1.76)$ \\
\hline No. of Quads & 40 & 3139 & 2201 & 2133 \\
\hline Max. Quad-Field (T) & 0.3 & 2.0 & 1.25 & 1.0 \\
\hline Max. Dipole-Field (T) & - & - & 0.75 & 1.01 \\
\hline \multicolumn{5}{|l|}{ Dimensionless paramaeters } \\
\hline$\sigma_{0}$ (degrees) & $78-44$ & $80-20$ & $90-18$ & $80-25$ \\
\hline$\sigma / \sigma_{0}$ & $0.20-0.27$ & $0.06-0.09$ & $0.43-0.20$ & $0.65-0.27$ \\
\hline$\dot{K}$ & $4.0-2.0 \times 10^{-4}$ & $9.0-0.9 \times 10^{-4}$ & $9.0-1.0 \times 10^{-5}$ & $11.0-3.5 \times 10^{-6}$ \\
\hline$\nu$ & $4.3-2.5$ & $92-22$ & $65-15$ & $63-20$ \\
\hline
\end{tabular}

${ }^{*}$ SR: Small Recirculator; LER, MER, HER: Low-, Medium-, and High-Energy Ring of C-Design LLNL/LBNL/FMT study (ref. 1). 
Table 2. Outline of design constraints of small recirculator

Practical considerations:

Circumference

Pipe radius

Beam energy and current

Ion species

Geometrical design considerations :

Pipe rad./max. beam rad.:

Length of quad

Length of dipoles

Quad \& dipole ends
$C \sim 14 \mathrm{~m}$ to fit in reasonable experimental area

$a \geq 3$ to $4 \mathrm{~cm}$ for ease of diagnostics and access

Energy $\sim .1 \mathrm{MeV}$, Current $\sim 5 \mathrm{~mA}$ similar to SBTE (Single Beam Transport Experiment) $A=39$ (Potassium), a known source used by LBNL

$r_{p} / a_{m a x} \geq 1.9$ to allow for misalignments, image forces, and momentum dispersion

$\geq 3 r_{p}$ to minimize non-linear fields

$\geq 3 r_{p}$

$1 r_{p}$ at each end for fringe fields, standoff, \& structure

Considerations to demonstrate driver relevance:

Undepressed phase advance

Depressed phase advance

Perveance

No. of betatron periods/lap

Lap time

Energy dynamic range

Bunch length compression $\sigma_{0} \leq 80^{\circ}=1.4 \mathrm{rad}$ for beam stability

$\sigma<<\sigma_{0}$ for space charge dominated beam

$K \sim 10^{-3}$ to $10^{-4}$ to be similar to LER of driver

$\nu>1$ to demonstrate resonance traversal

$t_{\text {lap }} \sim 20 \mu$ s to demonstrate control at high rep. rate

$E_{\text {final }} / E_{\text {initial }}>\sim 4$ (in MER factor is 20)

$l_{\text {final }} / l_{\text {initial }}<\sim 1 / 2$ (in HER factor is $1 / 3$ ) 